\title{
Quadratic Regularization Design for 3d Cylindrical PET
}

\author{
Hugo Shi and Jeffrey A. Fessler \\ Department of Electrical Engineering and Computer Science \\ University of Michigan \\ Email: hugoshi@umich.edu
}

\begin{abstract}
Statistical methods for tomographic image reconstruction lead to improved spatial resolution and noise properties in PET. Penalized-likelihood (PL) image reconstruction methods involve maximizing an objective function that is based on the log-likelihood of the sinogram measurements and on a roughness penalty function to control noise. In emission tomography, PL methods (and MAP methods) based on conventional quadratic regularization functions lead to nonuniform and anisotropic spatial resolution, even for idealized shift-invariant imaging systems. We have previously addressed this problem for parallel-beam 2D emission tomography [1], and for fan-beam 2D transmission tomography [2] by designing data-dependent, shift-variant regularizers that improve resolution uniformity and isotropy. even for idealized shift-invariant imaging systems. This paper extends those methods to 3D cylindrical PET, using an analytical design approach that is numerically efficient.
\end{abstract}

\section{INTRODUCTION}

Reconstructed images using Penalized-Likelihood (PL) methods or MAP methods exhibit non-uniform and anisotropic resolution as a result of interactions between the loglikelihood function and conventional quadratic regularizers. One could use a Quadratic Penalized Unweighted Least Squares (QPULS) method to avoid non-uniformities and anisotropy, however QPULS yields poor noise properties (similar to FBP). One can also use Maximum-Likelihood(ML) methods, and then post filter, however convergence of pure ML is extremely slow due to the ill-conditioned nature of the problem. We attempt to design quadratic penalties that will eliminate noise, improve the convergence over pure ML algorithms, and preserve uniformity and isotropy in the reconstructed images.

Previous work includes regularization design for parallel beam 2D PET systems and 2D fan-beam computer tomography [3], [2]. Qi and Leahy in [4] also proposed a regularization design method that provides a uniform peak amplitude of the local impulse response function for 3D PET, but that approach does not ensure isotropic resolution properties. This paper extends the 2D analysis of [3] to 3D cylindrical PET systems. section III discusses the derivation of the 3D approach and section $\mathrm{V}$ the results of our simulation.

\section{BACKGROUND}

We use PL estimation in which the object $\underline{x}$ is estimated by maximizing this objective function

$$
\begin{aligned}
& \Phi(\underline{y}, \underline{x})=L(\underline{y}, \underline{x})-\beta R(\underline{x}) \\
& L(\underline{y}, \underline{x})=\sum_{i} y_{i} \log \bar{y}_{i}(\underline{x})-\bar{y}_{i}(\underline{x}), \underline{y}=\mathbf{A} \underline{x}
\end{aligned}
$$

where $L$ is the log-likelihood, $\underline{y}$ is the data, $\underline{x}$ is the object, A is our system matrix, and $R$ is our quadratic regularizer. Traditional regularizers are of the form

$$
\sum_{j=1}^{P} \sum_{k=1}^{P} \frac{1}{2} r_{j k}\left(x_{j}-x_{k}\right)^{2}
$$

where $r_{j k}$ are user defined weights that allow different penalties for different pixel differences, $x_{j}$ is the $j$ th pixel value of an object $\underline{x}$, and $\underline{x}$ has $P$ pixels. We use the following expression to formulate our regularizer which preserves the same functionality as (1) but makes frequency domain analysis more intuitive. We define $R(x)$ as:

$$
\begin{aligned}
R(\underline{x}) & =\frac{1}{2} x^{\prime} \mathbf{R} x=\sum_{\underline{n}} \sum_{l=1} \frac{1}{2} r_{l}^{j}\left|\left(c_{l} * * * \underline{x}\right)(\underline{n})\right|^{2} \\
c_{l}[\underline{n}] & =\frac{1}{\|\underline{n}\|}\left(\delta[\underline{n}]-\delta\left[\underline{n}-\underline{n}_{l}\right]\right)
\end{aligned}
$$

Here $c_{l}$ is a function which when convolved with a volume takes the difference between a voxel and it's $l$ th neighbor. $r_{l}^{j}$ is a user defined weighting which weights differences between the $j$ th pixel and it's $l$ th neighbor.

The Local Impulse Response(LIR) for emission tomography with a Poisson distribution is [5]:

$$
l^{j}(\underline{x})=\left[\mathbf{A}^{\prime} \mathbf{W A}+\beta \mathbf{R}\right]^{-1} \mathbf{A}^{\prime} \mathbf{W} \mathbf{A} \delta_{j}
$$

where $\mathbf{W}=\operatorname{diag}\left[\frac{1}{\bar{y}_{i}(\underline{x})}\right]$. As is evident from (4), the LIR depends on our regularizer, $\mathbf{R}$. Our goal is to design $\mathbf{R}$ such that our LIR $l^{j}$ matches a target $l^{0}$ at every pixel $j$. Unfortunately, designing a spatially variant $\mathbf{R}$ so that $l^{j}$ matches $l^{0}$ is incredibly difficult. We look to the frequency domain to simplify this process.

Assuming $\mathbf{A}^{\prime} \mathbf{W A}$ and $\mathbf{R}$ are locally shift invariant, we can create a local frequency response for $\mathbf{A}^{\prime} \mathbf{W A}$ and $\mathbf{R}$ yielding the following local frequency response for our reconstruction system.

$$
L^{j}=\frac{F\left(\mathbf{A}^{\prime} \mathbf{W A} \delta_{j}\right)}{F\left(\mathbf{A}^{\prime} \mathbf{W} \mathbf{A} \delta_{j}\right)+\beta F\left(\mathbf{R} \delta_{j}\right)}
$$


where $F()$ denotes a Fourier transform. Taking the Fourier transform of (3), and then converting to spherical coordinates yields the following expression:

$$
\begin{aligned}
R(\varrho, \Phi, \Theta) & \approx(2 \pi \varrho)^{2} \sum_{l=1} r_{l}^{j}\left(e\left(\Phi_{l}, \Theta_{l}\right) \cdot e(\Phi, \Theta)\right)^{2} \\
e(\Phi, \Theta) & =(\cos \Theta \cos \Phi, \cos \Theta \sin \Phi, \sin \Theta)
\end{aligned}
$$

\section{3D REGULARIZATION DESIGN}

Assuming small acceptance angles, one can show using continuous space analogous that the local frequency response of $\mathbf{A}^{\prime} \mathbf{W A}$ is approximately

$$
F\left(\mathbf{A}^{\prime} \mathbf{W A} \delta_{j}\right)=\frac{w_{j}(\Phi)}{|\varrho| \cos (\Theta)} .
$$

In this equation, $w_{j}(\Phi)$ maps the weights from $\mathbf{W}$, which come from the sinogram, to the image domain. To find $w_{j}(\Phi)$ we backproject the weights from the projection at angle $\Phi$ over the image domain and find the value that corresponds to the $j$ th voxel. Next we must select our target frequency response in $3 \mathrm{~d}$. We use the LIR from unweighted, regularized reconstruction since it is uniform and mostly isotropic. This yields the following:

$$
\begin{aligned}
\frac{F\left(\mathbf{A}^{\prime} \mathbf{A} \delta_{j}\right)}{F\left(\mathbf{A}^{\prime} \mathbf{A} \delta_{j}\right)+F\left(\beta \mathbf{R}_{\mathbf{0}} \delta_{j}\right)} & \approx \frac{\frac{1}{|\varrho| \cos (\Theta)}}{\frac{1}{|\varrho| \cos (\Theta)}+F\left(\beta \mathbf{R}_{\mathbf{0}} \delta_{j}\right)} \\
& \approx \frac{1}{1+\beta \cos (\Theta)|\varrho| F\left(\mathbf{R}_{0} \delta_{j}\right)}
\end{aligned}
$$

This response is slightly anisotropic, due to the $\cos (\Theta)$ term in the denominator. To make it isotropic we choose $\mathbf{R}_{0}=\frac{|2 \pi \varrho|^{2}}{\cos (\Theta)}$, yielding

$$
L^{0}=\frac{1}{1+\beta(2 \pi)^{2}|\varrho|^{3}} .
$$

Now, by choosing $r_{l}^{j}$, we design $\mathbf{R}$ to make the actual LIR close to our target LIR,

$$
\frac{w^{j}(\Phi)}{w^{j}(\Phi)+\beta|\varrho| \cos (\Theta) R(\varrho, \Phi, \Theta)} \approx \frac{1}{1+\beta(2 \pi)^{2}|\varrho|^{3}} .
$$

After cross multiplying and simplifying, we use a NonNegative Least Squares(NNLS) algorithm to solve the following minimization problem for $r_{l}^{j}$

$$
\begin{aligned}
\underline{r}^{j}= & \underset{r \geq 0}{\arg \min } \int_{0}^{\pi} \int_{-\pi / 2}^{\pi / 2} \mid w^{j}(\Phi)- \\
& \left.\sum_{l=1}^{L} r_{l}^{j} \cos (\Theta)\left(e\left(\Phi_{l}, \Theta_{l}\right) \cdot e(\Phi, \Theta)\right)^{2}\right|^{2} d \Theta, d \Phi
\end{aligned}
$$

\section{IMPLEMENTATION DETAILS}

We can break up the term $\sum_{l=1}^{L} r_{l}^{j} \cos (\Theta)\left(e\left(\Phi_{l}, \Theta_{l}\right)\right.$. $e(\Phi, \Theta))^{2}$ as $\mathbf{B C} \underline{r}^{j}$ where $\underline{r}^{j}$ is a $L \times 1$ vector of penalty coefficients. We can expand $\cos (\Theta)\left(e\left(\Phi_{l}, \Theta_{l}\right) \cdot e(\Phi, \Theta)\right)^{2}$ into 6 orthonormal basis functions which will make up the columns of $\mathbf{B}$. $\mathbf{C}$ is a matrix of linear combinations coefficients such that $\sum_{l=1} r_{l}^{j} \cos (\Theta)\left(e\left(\Phi_{l}, \Theta_{l}\right) \cdot e(\Phi, \Theta)\right)^{2}=\mathbf{B C} r$. We undergo this factorization to simplify the problem. Our current problem is framed as

$$
\underline{r}^{j}=\underset{\underline{r}^{j} \geq 0}{\arg \min }\left\|w-\mathbf{B C} \underline{r}^{j}\right\|^{2}
$$

This is equivalent too

$$
\begin{aligned}
& =\underset{\underline{r}^{j} \geq 0}{\arg \min }\left\|w-\mathbf{B C} \underline{r}^{j}\right\|^{2} \\
& =\underset{\underline{r}^{j} \geq 0}{\arg \min }<w-\mathbf{B C} \underline{r}^{j}, w-\mathbf{B C} \underline{r}^{j}> \\
& =\underset{\underline{r}^{j} \geq 0}{\arg \min }\|w\|^{2}-2<w, \mathbf{B C} \underline{r}^{j}>+<\mathbf{B C} \underline{r}^{j}, \mathbf{B C} \underline{r}^{j}> \\
& \quad \underset{\underline{r}^{j} \geq 0}{\arg \min }\|w\|^{2}-2<\mathbf{B}^{*} w, \mathbf{C} \underline{r}^{j}> \\
& \quad+<\mathbf{B}^{*} \mathbf{B C} \underline{r}^{j}, \mathbf{C} \underline{r}^{j}>
\end{aligned}
$$

Since the columns of $\mathbf{B}$ are orthonormal functions, $\mathbf{B}^{*} \mathbf{B}$ is the Identity matrix. We can replace $\|w\|^{2}$ with $\left\|\mathbf{B}^{*} w\right\|^{2}$ since it is an irrelevant constant which has no effect on our minimization, leaving us with

$$
\begin{aligned}
& =\underset{\underline{r}^{j} \geq 0}{\arg \min }\left\|\mathbf{B}^{*} w\right\|^{2}-2<\mathbf{B}^{*} w, \mathbf{C} \underline{r}^{j}>+\left\|\mathbf{C} \underline{r}^{j}\right\|^{2} \\
& =\underset{\underline{r}^{j} \geq 0}{\arg \min }\left\|\mathbf{B}^{*} w-\mathbf{C} \underline{r}^{j}\right\|^{2}
\end{aligned}
$$

$\mathbf{B}^{*} w$ produces a $6 \times 1$ vector, and $\mathbf{C}$ is $6 \times L$, making this a much smaller minimization problem.

\section{A. Problems with NNLS}

In the 2D case, one can minimize (9) analytically [3] such that the solution is minimum norm. In a NNLS problem that is underdetermined there are many solutions that minimize the cost function. Finding the solution with a minimum norm solves for $\underline{r}^{j}$ as a continuous function of $B^{*} w$ and leads to good image reconstruction properties. Without the continuous mapping from $B^{*} w$ to $\underline{r}^{j}$, neighboring pixels can have drastically different weights, thus violating our assumptions of local spatial invariance. [3] exploits properties of the matrices used to solve the $2 \mathrm{D}$ problem to find a minimum norm solution. Unfortunately those properties do not hold in the 3D case. To compensate for this problem, we try to alter the minimization problem from arg $\min _{r^{j} \geq 0}\left\|\mathbf{B}^{*} w-\mathbf{C} \underline{r}^{j}\right\|^{2}$ to $\arg \min _{\underline{r}^{j} \geq 0}\left\|\mathbf{B}^{*} w-\mathbf{C} \underline{r}^{j}\right\|^{2}+\epsilon|| \underline{r}^{j} \|^{2}$ so that the norm of $\underline{r}^{j}$ becomes a factor in the cost function. We append a scaled identity matrix to the bottom of $\mathbf{C}$ and zero $\operatorname{pad} \mathbf{B}^{H} w$

$$
\tilde{\mathbf{C}}=\left(\begin{array}{c}
\mathbf{C} \\
\epsilon \mathbf{I}
\end{array}\right), \tilde{d}=\left(\begin{array}{c}
\mathbf{B}^{H} w \\
\underline{\mathbf{o}}
\end{array}\right)
$$

so that (9) is changed to

$$
\begin{aligned}
\underline{r}^{j} & =\underset{\underline{r}^{j} \geq 0}{\arg \min }\left\|\underline{\tilde{d}}-\tilde{\mathbf{C}} \underline{r}^{j}\right\| \\
& =\underset{\underline{r}^{j} \geq 0}{\arg \min }\left\|\mathbf{B}^{*} w-\mathbf{C} \underline{r}^{j}\right\|^{2}+\epsilon\left\|\underline{r}^{j}\right\|^{2}
\end{aligned}
$$

This fix eliminates the discontinuities. 


\section{B. Convergence}

Since we are minimizing with a non-negative constraint, our design has the potential to yield many $r_{l}^{j}$ values that are zero. If there are too many zeroes in $r_{l}^{j}$, there will be zeroes in the hessian, leaving us with bad convergence properties. Instead of using a non-negative constraint, we would like to have $r_{l}^{j}$ be greater than $\epsilon$ for selected $r_{l}^{j}$ to ensure that enough $r_{l}^{j}$ are non-zero. We select the 3 adjacent neighbors in the immediate $\mathrm{x}, \mathrm{y}$, and $\mathrm{z}$ directions to be non-zero. We turn to previous work to select $\epsilon$. In [5], Fessler derives a spatially variant $\beta$ which seeks to preserve uniform spatial resolution. So for any given pixel, we take $\epsilon=\alpha \beta_{j}^{2}$ where this $\beta_{j}$ is the spatially variant $\beta$ for a pixel $\left(x_{j}, y_{j}, z_{j}\right)$. Increasing $\alpha$ improves convergence at the expense of isotropy, while use of the spatially variant $\beta$ helps us preserve uniformity.

Now we must formulate our problem so that NNLS algorithms will accept this new constraint. We can create a vector $\underline{\epsilon}$ which is zero for most neighbors, and $\alpha \beta_{j}^{2}$ for immediate $\mathrm{x}$, $\mathrm{y}$ and $\mathrm{z}$ neighbors. let $\underline{\tilde{r}}^{j}=\underline{r}^{j}-\underline{\epsilon}$. Solving with the constraint of $\underline{\tilde{r}}^{j} \geq 0$ ensures that $\underline{r}^{j} \geq \underline{\epsilon}$. Plugging $\underline{\tilde{r}}^{j}$ into (11) we get $\left|\tilde{\mathbf{C}} \underline{\tilde{r}}^{j}-\underline{\tilde{d}}\right|=\left|\tilde{\mathbf{C}}\left(\underline{r}^{j}-\underline{\epsilon}\right)-\underline{\tilde{d}}\right|=\left|\tilde{\mathbf{C}} \underline{r}^{j}-(\underline{\tilde{d}}+\tilde{\mathbf{C}} \underline{\epsilon})\right|$ which can be plugged into an NNLS algorithm. Using $\underline{\tilde{r}}^{j}$ for reconstruction yields positive results, discussed in the next section.

\section{RESUlTs}

We simulated a 3D PET system that images a 400mm x $400 \mathrm{~mm} \times 164 \mathrm{~mm}$ volume. The simulated imaging system has 143 rays per view spaced $4 \mathrm{~mm}$ apart, 80 evenly spaced view angles rotated along $\Phi$, and 5 cross plane angles for $\Theta$. The reconstructed images consist of $100 \times 100 \times 414 \mathrm{~mm}$ pixels. We choose a target spatial resolution with a 2 pixel FWHM, or equivalently $4 \mathrm{~mm}$. The following images show cross sections of $3 \mathrm{~d}$ Point Spread Functions (PSF) at different locations, showing the $\mathrm{xy}$ slice, $\mathrm{xz}$ slice, and $\mathrm{yz}$ slice from top to bottom. From left to right, are the PSFs at $(25,25,0)$, $(15,15,0),(15,15,15)$, and $(25,0,0)$. PSFs from conventional regularization are shown in figure 1. PSFs from our proposed regularization scheme are shown in figure 2. Looking at these images, one may observe a great deal of anisotropy in the PSFs created from conventional regularization. The xy slices are stretched out in different directions depending on where the pixel lies with respect to the center of the image. The $x z$ and yz slices all seem flatter and smaller when compared to xy slices. Looking at the PSFs generated using our proposed regularization scheme, each slice looks more isotropic, and each slice looks to be about the same size. The flatness of $\mathrm{xz}$ and yz slices has been removed, and the xy slices are no longer stretched. The pixel at $(25,25,0)$ has not been completely corrected though, it's xy slice appears to be boxy with tails, instead of looking round, and the $\mathrm{xz}$ and $\mathrm{yz}$ slices are not completely isotropic. This shows the limitations of this method in approximating $w^{j}(\Phi)$.

In the next few figures, we take $x y, x z$, and yz slices of the PSFs, and then measure the FWHM cutting through the center of the PSF at different angles to get a sense of isotropy. The

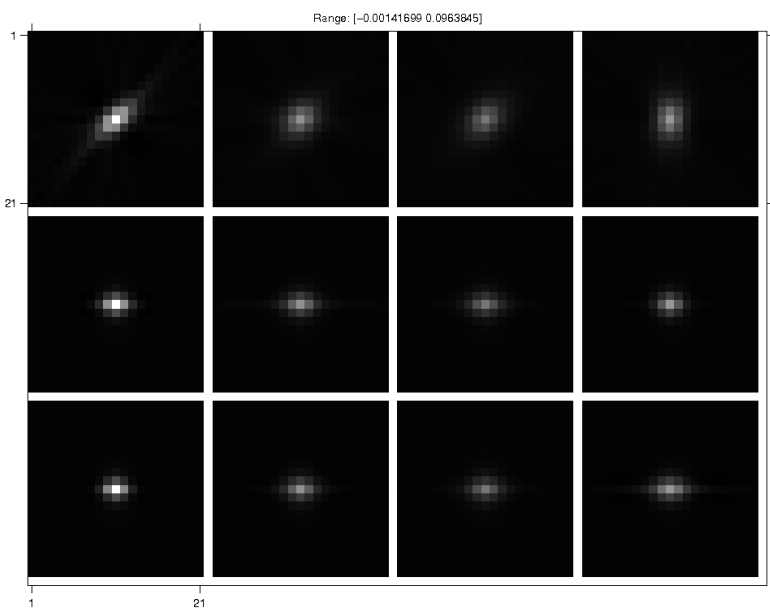

Fig. 1. $x y, x z$, and $y z$ (top to bottom) slices of PSFs created using conventional regularization at $(25,25,0),(15,15,0),(15,15,15)$, and $(25,0,0)$ (left to right).

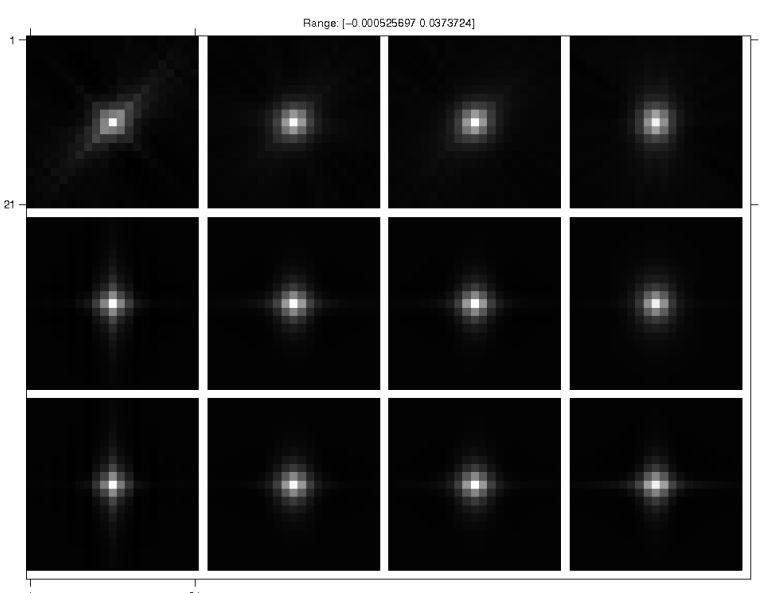

Fig. 2. $x y, x z$, and $y z$ (top to bottom) slices of PSFs created using our proposed regularization at $(25,25,0),(15,15,0),(15,15,15)$, and $(25,0,0)$ (left to right).

uniformity of these plots are a rough measure of the isotropy of these impulse functions. Figure 3 and 4 shows plots of the FWHM for PSFs at $(25,25,0)$ and $(15,15,15)$.

Tables I displays maximum and minimum FWHMs for PSFs at $(25,25,0)$ and $(15,15,15)$ allowing for a more quantitative assessment of isotropy. Even for $(25,25,0)$ which exhibits less improvement than $(15,15,15)$, there is less variation in FWHM for PSFs generated using our proposed regularization scheme than PSFs generated using conventional regularization.

We simulated a noiseless sinogram using our true image which consists of 2 spherical shells of uniform intensity inside a cylinder. For the following figures we will be showing xy slice 21 of the volume, followed by $\mathrm{xz}$ slice 41 , and then yz slices 41 . We reconstructed volumes using two methods. Volumes reconstructed using conventional regularization are shown in figure 7 and 8 . Volumes reconstructed using our proposed regularization design are shown in figures 5 and 6 . Volumes reconstructed using our proposed regularization 

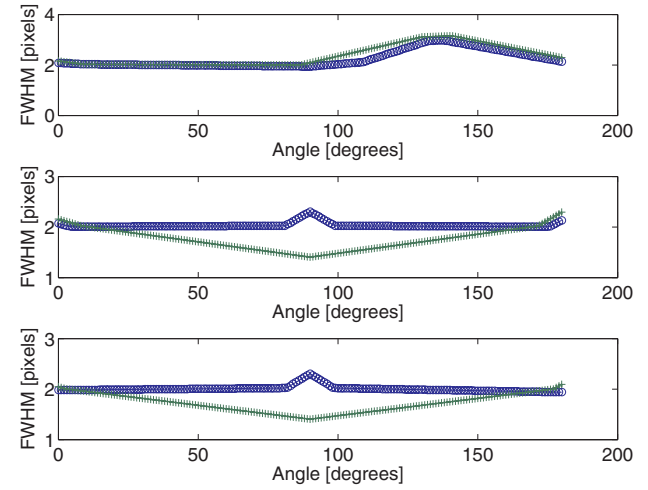

Fig. 3. FWHM plots for PSF at $(25,25,0)$. Proposed regularization marked with circles, conventional marked with pluses. $x y, x z, y z$ slices from top to bottom
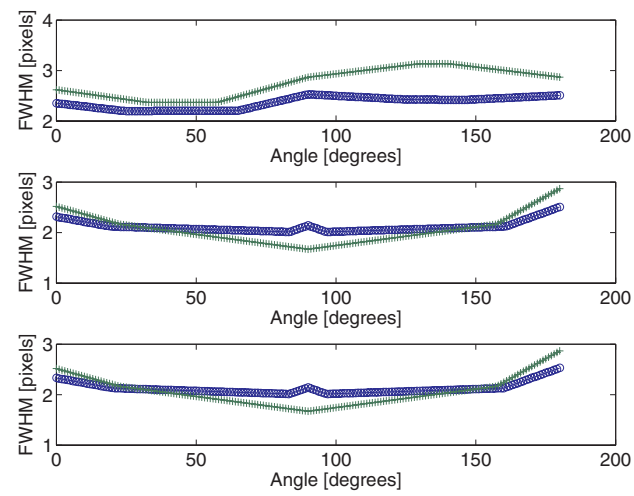

Fig. 4. FWHM plots for PSF at $(15,15,15)$. Proposed regularization on the left, Conventional on the right. xy, xz, yz slices from top to bottom

design show greater uniformity than those reconstructed using conventional regularization. However, due to errors in approximating $w^{j}(\Phi)$ which results in anisotropic impulse responses with tails as shown for pixel $(25,25,0)$ in figure 2 , there are streak like artifacts in the xy slice.

We selected the sphere centered at $(26,0,0)$ and cut an $x y, x z$, and yz profile through it for volumes reconstructed using our proposed and the conventional regularization scheme. These are shown in figures 9, 10, and 11. These figures show a significant decrease in the variation of intensity around the uniform ring for volumes reconstructed using our proposed regularization scheme.

\section{CONCLUSION}

This paper has summarized an extension of previous Fourier-based regularization design [3] to the $3 \mathrm{~d}$ cylindrical case. Simulation results show that the method improves isotropy and uniformity of spatial resolution properties compared to conventional quadratic regularization schemes. Unfortunately simulation results also show existence of streaklike artifacts at the edges of the image from our conventional regularization scheme. In the future, we would like to develop a sort of hybrid scheme between the proposed regularization design and conventional regularization which would preserve
TABLE I

MAXIMUM AND Minimum FWHM VALUES FOR 2 PSFs

\begin{tabular}{|c|c|c|c|c|}
\hline \multirow[t]{2}{*}{$(25,25,0)$} & \multicolumn{2}{|c|}{ Proposed } & \multicolumn{2}{|c|}{ Conventional } \\
\hline & $\min$ & $\max$ & $\min$ & $\max$ \\
\hline xy & 1.9387 & 2.9770 & 2.0035 & 3.1553 \\
\hline $\mathrm{xz}$ & 2.0061 & 2.3060 & 1.4048 & 2.2946 \\
\hline $\mathrm{yz}$ & 1.9387 & 2.3060 & 1.4048 & 2.0932 \\
\hline \multirow[t]{2}{*}{$(15,15,15)$} & \multicolumn{2}{|c|}{ Proposed } & \multicolumn{2}{|c|}{ Conventional } \\
\hline & $\min$ & $\max$ & $\min$ & $\max$ \\
\hline xy & 2.1954 & 2.5281 & 2.3689 & 3.1347 \\
\hline $\mathrm{xz}$ & 2.0137 & 2.5069 & 1.6706 & 2.8698 \\
\hline $\mathrm{yz}$ & 2.0141 & 2.5281 & 1.6706 & 2.8676 \\
\hline
\end{tabular}

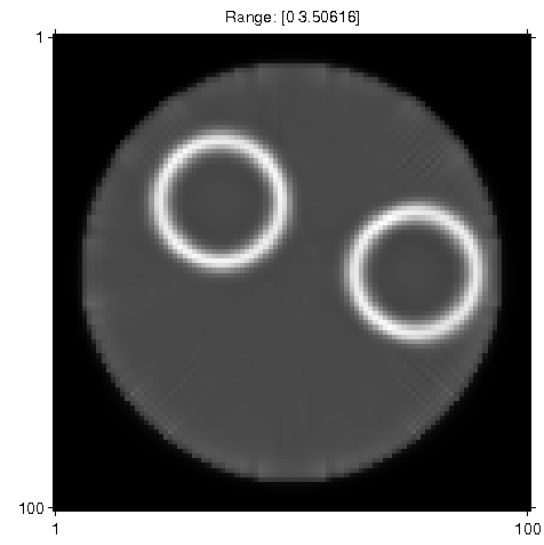

Fig. 5. Volume reconstructed with proposed regularization design. xy slice
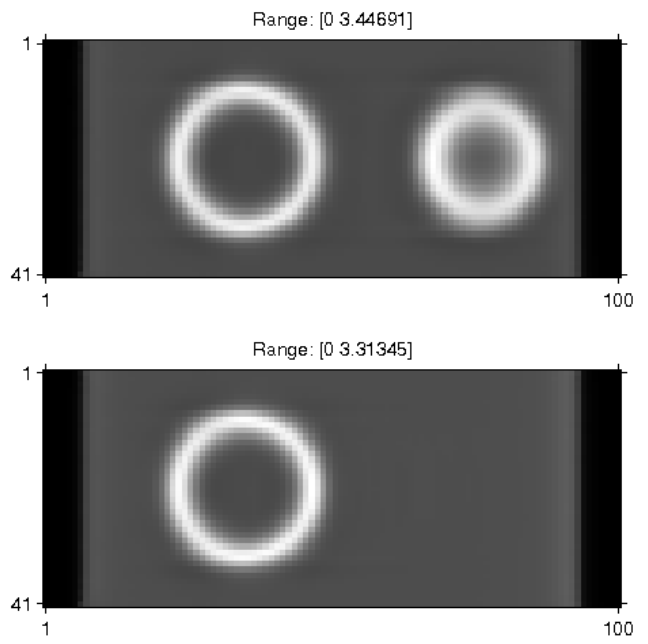

Fig. 6. Volume reconstructed with proposed regularization design. xz slice then yz slice 


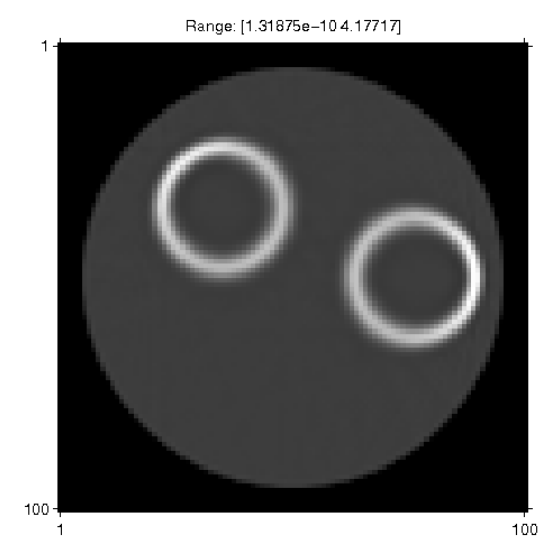

Fig. 7. Volume reconstructed with conventional regularization design. xy slice
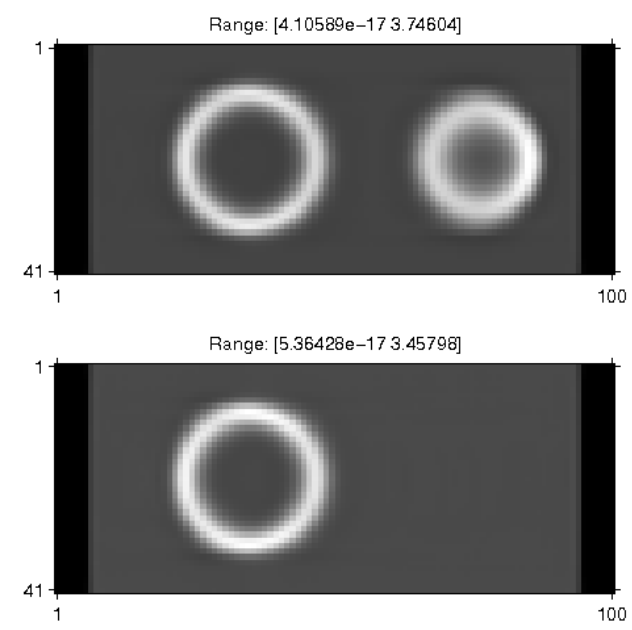

Fig. 8. Volume reconstructed with conventional regularization design. $\mathrm{xz}$ slice then yz slice

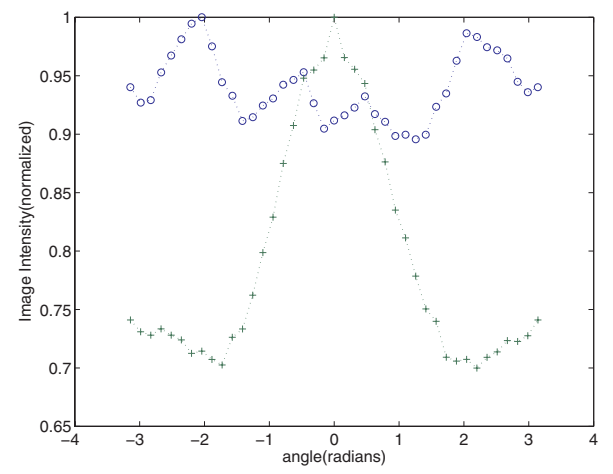

Fig. 9. xy profile of sphere. Circles represent our proposed scheme, pluses represent the conventional scheme.

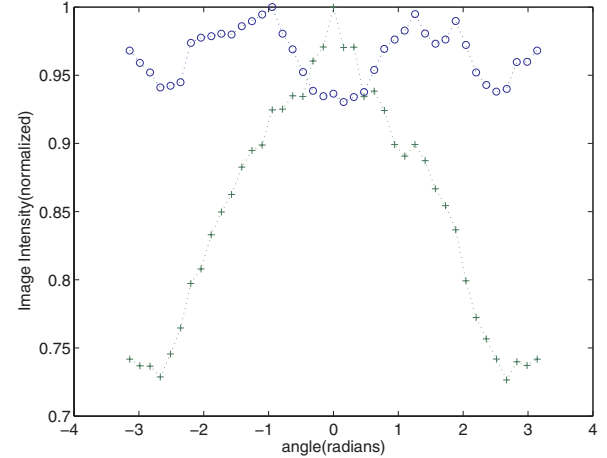

Fig. 10. xz profile of sphere. Circles represent our proposed scheme, pluses represent the conventional scheme.

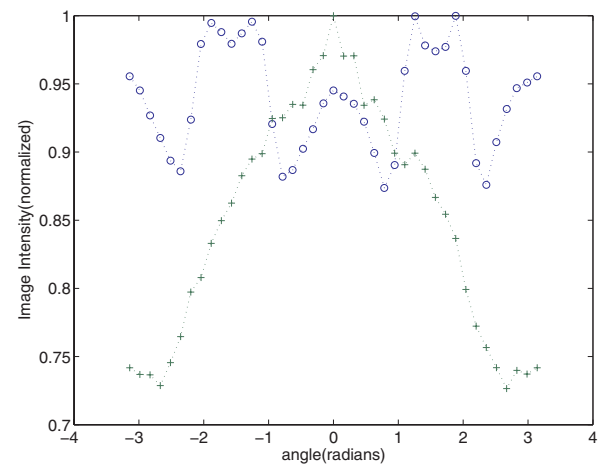

Fig. 11. yz profile of sphere. Circles represent our proposed scheme, pluses represent the conventional scheme.

uniform and isotropic spatial resolution and eliminate the streak-like artifacts. We would also like to analyze the convergence properties of PL algorithms using these penalties, as well as work on ways of speeding up the computation of $r_{l}^{j}$. Future work also includes generalization to spatially variant blur, 3D extensions to cone beam CT, and application to real PET data.

\section{ACKNOWLEDGMENT}

Work supported in part by NIH grants CA-87634, and DOE grant DE-FG02-87ER60561.

\section{REFERENCES}

[1] J. W. Stayman and J. A. Fessler, "Regularization for uniform spatial resolution properties in penalized-likelihood PET reconstruction," in Biomedical Imaging: Beyond Diagnostics, 1999, p. A4.

[2] H. Shi and J. A. Fessler, "Quadratic regularization design for fan beam transmission tomography," in Proc. SPIE 5747, Medical Imaging 2005: Image Proc., 2005, pp. 2023-33.

[3] J. A. Fessler, "Analytical approach to regularization design for isotropic spatial resolution," in Proc. IEEE Nuc. Sci. Symp. Med. Im. Conf., vol. 3, 2003, pp. 2022-6.

[4] J. Qi and R. M. Leahy, "Resolution and noise properties of MAP reconstruction for fully 3D PET," IEEE Trans. Med. Imag., vol. 19, no. 5, pp. 493-506, May 2000.

[5] J. A. Fessler and W. L. Rogers, "Spatial resolution properties of penalizedlikelihood image reconstruction methods: Space-invariant tomographs," IEEE Trans. Im. Proc., vol. 5, no. 9, pp. 1346-58, Sept. 1996. 